\title{
Harmonics Optical Biopsy of Human Skin
}

\author{
Shih-Peng Tai ${ }^{1}$, Tsung-Han Tsai ${ }^{1}$, Wen-Jeng Lee ${ }^{2,3}$, Shi-Wei Chu' ${ }^{1}$, Hsin-Yi Huang ${ }^{4}$, Yi-Hua Liao $^{5}$, and \\ Chi-Kung Sun ${ }^{1}$ \\ 1. Graduate Institute of Electro-Optical Engineering, National Taiwan University, Taipei 10617, TAIWAN \\ PhoneD886-2-23659703, FAXD886-2-23677467,E-mail口 d92941004@ntu.edu.tw \\ 2. Graduate Institute of Electrical Engineering. National Tatwan University, Taipei 10617, TAIWAN \\ 3. Department of Medical Imaging, National Taiwan University Hospital, Taipei J0617, Taiwan \\ 4. Department of Pathology, National Taiwan University Hospital, Taipei 10617, Taiwan \\ 5. Department of Dermatology, National Taiwan University Hospital, Taipei 10617, Taiwan
}

\begin{abstract}
Avoiding the in-focus photodamage and phototoxicity problem of two-photonfluorescence excitation, noninvasive harmonics optical biopsy of human skin is demonstrated based on a $1200-1300-\mathrm{nm}$ femtosecond light source.

(C) 2004 Optical Society of America

OCIS codes: (170.1870) Dermatology; (180.6900) Three-dimensional microscopy; (180.5810) Scanning microscopy;

(190.4160) Mutharmonic generation.
\end{abstract}

Traditional biopsy requires the removal, fixation, and staining of tissues from the human body. Its procedure is invasive and painful. Non-invasive in vivo optical biopsy is thus required, which should provide non-invasive, highly penetrative, three-dimensional (3D) imaging with sub-micron spatial resolution. Optical biopsy based on scanning two-photon fluorescence microscopy (TPFM) $[1,2]$ is a good method for biopsy of skin due to its high lateral resolution, low out-of-focus damage, and intrinsic three-dimensional (3D) section capability. Previous two-photon fluorescence biopsy of skin $[1,2]$ based on a $780 \mathrm{~nm}$ femtosecond light provides high resolution imaging from the skin surface through the epidermal-dermal junction. However for future clinical applications without surgery, current $700-850 \mathrm{~nm}$ based laser scanning technology still presents several limitations including low penetration depth, in-focus cell damages, and multi-photon phototoxicity due to high optical intensity in the $800 \mathrm{~nm}$ wavelength region, and toxicity if exogenous fluorescence markers were required.

Here we study the harmonics optical biopsy of a human skin sample using a femtosecond Cr:forsterite laser cenetered at $1230 \mathrm{~nm}$ [3]. According to previous studies, light attenuation (including both absorption and scattering) in human skin [4] reaches a minimum around $1200-1300 \mathrm{~nm}$ due to the combination of diminishing scattering cross-section with wavelength and avoiding the resonant molecular absorption of common tissue constituents such as water, melanin, and hemoglobin. Besides, moving the excitation wavelength longer than $1200 \mathrm{~nm}$ makes second harmonic generation (SHG) and third harmonic generation (THG) possible due to visible SHG and THG wavelength that can avoid strong UV region absorption in human-tissues. Higher harmonics generation is known to leave no energy deposition to the interacted matters due to their energyconservation characteristic. This energy-conservation characteristic provides the "noninvasive" nature desirable for clinical imaging. Due to its nonlinear nature, the generated SHG and THG intensities depend on square and cubic of the incident light intensity. Similar to multi-photon induced fluorescence process, this nonlinear dependency allows localized excitation and is ideal for intrinsic optical sectioning in optical biopsy applications.

The study of the harmonics optical biopsy (HOB) of human skin was performed using a femtosecond $\mathrm{Cr}$ :forsterite laser centered at $1230 \mathrm{~nm}$ with $140 \mathrm{fs}$ pulsewidth at a $110 \mathrm{MHz}$ repetition rate. The thickness of the human skin sample was $\sim 1 \mathrm{~mm}$. Figure 1 shows example vertically sectioned $(x-z)$ HOB images taken from the human epidermis and dermis corresponding to forward propagating multi-harmonic generation intensities. From the vertically sectioned $\mathrm{HOB}$ images, the general histological structures in both epidermis and dermis layers can be identified through the THG modality due to its sensitivity to local optical inhomogeneity [5-7]. For example, the coverglass-formalin interface, stratum comeum, and basal layer can all be easily picked up through the THG modality (for example, see Fig.1 (b)). SHG, on the other hand, mainly reflects the distribution of connected tissues in the dermis layer (bottom of Fig. 1(a)) due to strong SHG generation in collagen fibers [8-10]. Through the SHG image (for example, see Fig.1 (a)), other structures like the keratin layer in the epidermis and the capillaries surrounded by connected tissues can also be clearly identified.

Figure 2 shows a horizontally sectioned ( $x-y$ ) HOB image (THG modality) taken from the basal layer inside a normal human skin at a depth of $90 \mu \mathrm{m}$ from the skin surface. Detailed sub-cellular structures in basal cells can be clearly resolved through THG. Figure 3 shows another horizontally sectioned HOB image (SHG modality) taken from the dermis layer at a depth of $136 \mu \mathrm{m}$ from the skin surface. While SHG displays the rich collagen fibril structures in the dermal layer, other histological structures like capillaries can also be identified (also revealed in THG image, not shown). More HOB images in human skin showing different histological structures, including blood cells, will be presented in this talk.

In summary, current $800 \mathrm{~nm}$ based two-photon optical biopsy technology still presents several limitations including finite penetration depth, in-focus cell damages, and phototoxicity due to the required high optical intensity and multi-photon absorption processes. Harmonics generation, however, can provide a truly non- 


\section{CThS4}

invasive biopsy tool with excel'erit three-dimensional spatial resolution due to their virtual transition and nonlinear characteristics. Combining with a 1200-1300-nm light source, our preliminary study suggests that this technique could provide sub-micron resolution deep-tissue non-invasive biopsy images in human skins without using fluorescence and exogenous markers. By improving the detection geometry (backward propagating), this technique could be useful for non-invasive clinical biopsy of human skin diseases and even useful for the early diagnosis of skin cancer symptom such as the angiogenesis. This work is sponsored by National Health Research Institute of Taiwan, R.O.C. through grant NHRI-EX92-9201EI.
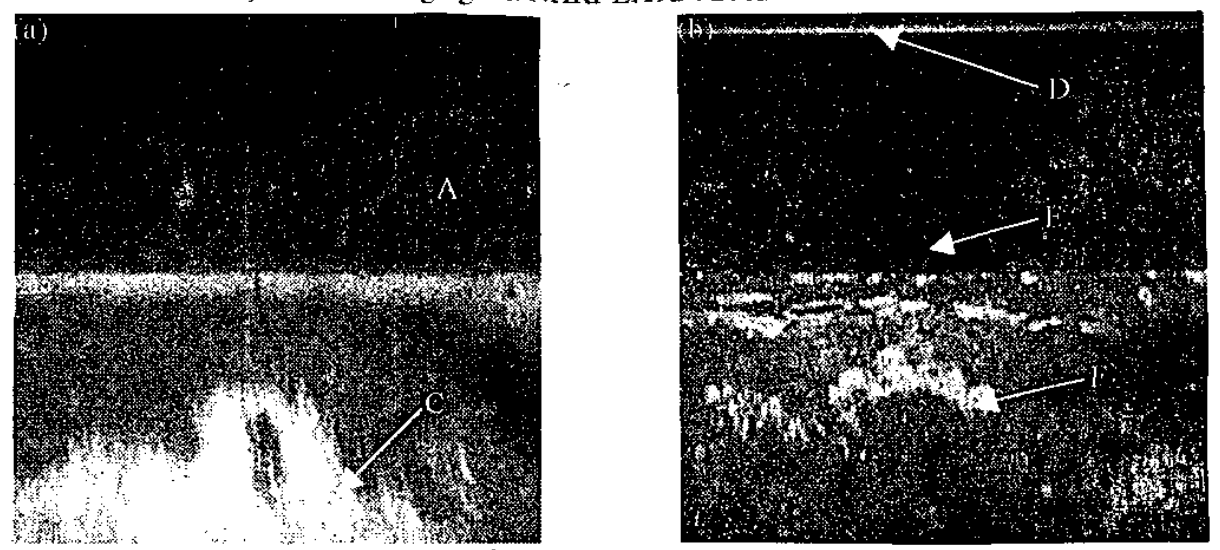

Fig. 1. Vertically sectioned HOB images taken from the human epidermis and dermis corresponding to emitted (a) SHG and (b) THG. Image size: $240 \times 380 \mu \mathrm{m}$. In the figures, A-F are keratin layer, capillary, connective tissue, coverglass-formalin interface, stratum corneum, and basal layer.

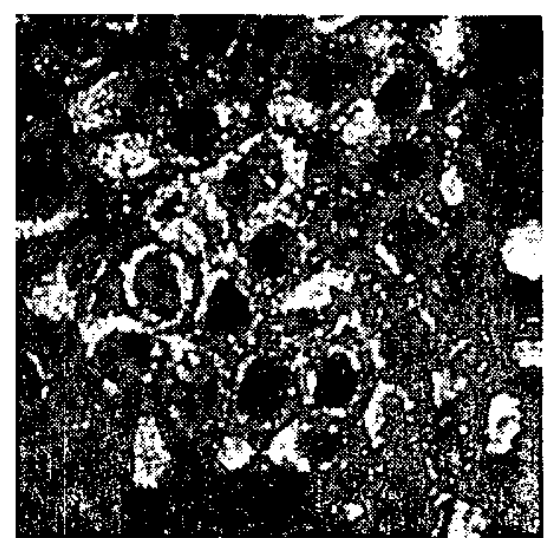

Fig. 2. Horizontally sectioned HOB image from the basal layer in the dermo-epidermal junction corresponding to the forward propagating THG modality. Image size: $60 \times 60 \mu \mathrm{m}$

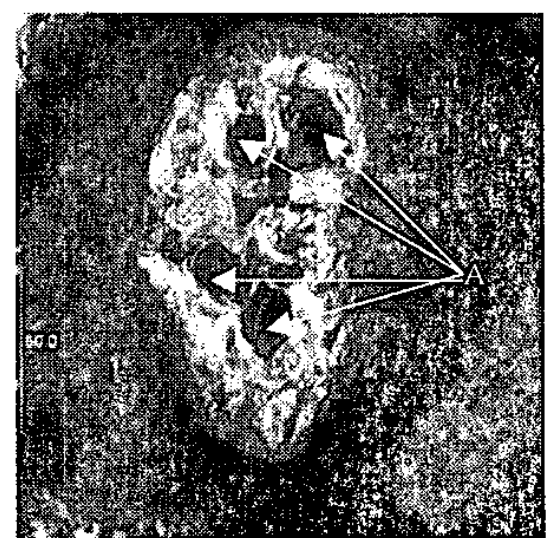

Fig. 3. Horizontally sectioned HOB image from the dermal layer corresponding to the forward propagating SHG 


\section{CThS4}

modality. Capillaries (A) surrounded by connected tissues can be clearly identified. Image size: $240 \times 240$ $\mu \mathrm{m}$.

\section{References}

[1] P. T. C. So, H.r Kim, and I. E. Kochevar, "Two photon deep tissue ex vivo imaging of mouse dermal and subcutaneous structures," Optics Express 3, 339 (1998).

[2] B. R. Masters and P. T. C. So, "Confocal microscopy and multi-photon excitation microscopy of human skin in vivo," Optics Express 8, 2 (2001).

[3] T.-M. Liu, S.-W. Chu, C.-K. Sun, B.-L. Lin, P. C. Cheng, and I. Johnson, "Multi-photon scanning microscopy using a Femtosecond Cr:forsterite laser," Scanning 23, 249 (2001).

[4] R. R. Anderson and J. A. Parish, "The optics of human skin," Journal of Investigative Dermatology 77, 13 (1981)

[5] S.-W. Chu, I-H. Chen, T.-M. Iiu, P. C. Cheng, C.-K. Sun, and B.-L. Lin, "Multimodal nonlinear spectral microscopy based on a femtosecond Cr:forsterite laser," Opt. Lett. 26, 1909 (2001).

[6] D. Yelin and Y. Silberberg, "Laser scanning third-harmonic-generation microscopy in biology,"Opt. Express 5, 169(1999)

[7] J. A. Squier, M. Muller, G. J. Brakenhoff, and K. R. Wilson, "Third harmonic generation microscopy," Opt. Express 3, 315 (1999).

[8] Y. Guo, P. P. Ho, H. Savage, D. Harris, P. Sacks, and S. Schantz, F. Liu, N. Zhadin, and R. R. Alfano, "Second-harmonic tomography of tissues," Opt. Lett. 22, 1323 (1997).

[9] P. J. Campänola, A. C Millard, M. Terasaki, P. E. Hoppe, C. J. Malone, and W. A. 12 Mohler, "Threedimensional high-resolution second-harmonic generation imaging of endogenous structural proteins in biological tissues," Biophy. J. 81, 493 (2002).

[10] S.-W. Chu, I-S. Chen, T.-M. Liu, C.-K. Sun, S.-P. Lee, B.-L. Lin, P.-C. Cheng, M.-X. Kuo, D.-J. Lin, and H.-L. Liu, "Nonlinear Bio-photonic Crystal Effects Revealed with Multi-modal Nonlinear Microscopy," $J$. Microscopy, 208, Part 3, 190 (2002). 